\title{
Pulsed Green Laser Induced Regular Stripes on Kerf Sidewall of Sapphire Substrate
}

\author{
Jiang Wei, Xie Xiaozhu*, Che Ronghong, Wei Xin, Hu Wei, Ren Qinglei,Weng Qing \\ School of Eletromechanical Engineering, Guangdong University of Technology, Guangzhou, \\ Guangdong 510006, China \\ E-mail: xiaozhuxie@gdut.edu.cn
}

\begin{abstract}
Pulsed green laser with wavelength of $532 \mathrm{~nm}$ is employed to dice the sapphire substrate. The regular periodical stripes are generated on the sidewall of the sapphire under the laser ablated groove after breaking. The result suggests the formation of the periodical stripes on the sidewall is due to the multiple thermal accumulation and thermal shocks during laser dicing. Laser processing parameters (laser pulse energy, scanning velocity and laser pulse repetition rate) affect the stripe space, length and shape. The laser pulse energy and scanning velocity are the two main influence factors, while the laser pulse energy is the least influence one. The appearance of stripes can deepen the laser-cut kerf depth and contribute to the sapphire breaking procedure after laser dicing.
\end{abstract}

DOI: 10.2961/jlmn.2017.01.0008

Keywords: pulsed green laser, sapphire substrate, regular periodical stripe, thermal stress, micromachining

\section{Introduction}

Sapphire with a wide band-gap of $\sim 8.8 \mathrm{eV}$ is of a particular technological interest since its basic optical, mechanical and thermo-physical properties. It is well suited for many applications, such as the substrates for the epitaxial growth of semiconductor film, wear resistant bearing and window for infrared equipment and high power lasers. However, with the properties of high thermal conductivity, high elastic modulus and high hardness, traditional methods, such as the etching (dry and wet) and the diamond blade dicing, are increasingly difficult to process the sapphire substrate due to their drawbacks [1.2].

Laser micromachining is a non-contact process without the problems of mechanical damage and tool wear, and it has advantages of narrow kerf, smooth section, high dicing velocity and high automation etc. Currently laser is widely used in metals, ceramics, glass and polymeric materials micro- and nano-fabrication [3-6]. Meanwhile, as the staggering growth of semiconductor industry, especially the LED industry and smart phone, laser dicing of silicon, glass and sapphire gradually appeals to several researchers [7-9]. As a result, laser-induced damage of solids has been of scientific and technological interest in transparent solids and the interaction of laser pulses with wide band gap materials has also been studied [10-12].

Direct laser inducing sidewall stripes or structures on crisp and hard material have been explored by nanosecond, picosecond and femtosecond lasers. Zhang [7] used a $355 \mathrm{~nm}$ nanosecond pulse laser to scribe the sapphire and found regular stripes are induced on the sidewall of sapphire. The regular stripes are proved to have improvement on light extraction efficiency (LEE) of InGaN-based lightemitting diodes. The similar phenomenon also occurs on hardened glass sidewall ablated by picosecond and femtosecond laser and the morphologies of the stripes vary with pulse duration, pulse energy and pulse numbers [13]. When a 1064nm nanosecond laser is focused into the interior of a silicon wafer and scanned in the horizontal direction, a high dislocation density layer and internal cracks are formed in the wafer [14]. Recently, regular stripes are found on the sidewall of the sapphire substrate by pulsed green laser in our previous research. The formation mechanism of the green laser induced stripes on sapphire sidewall is still unclear. The stripes size and morphologies affected by laser processing parameters are need to be exploited.

In the present paper, periodical stripes are achieved by fabricating kerfs on the sapphire substrate by a $532 \mathrm{~nm}$ DPSS nanosecond laser. Optical microscope and SEM are used to observe the morphologies of laser induced stripes on sapphire substrate. The formation of this regular stripe is explained according to the observed data and sapphire material features. The relationship between micro-inner morphology characteristics of the laser processed sapphire and the laser processing parameters is also analyzed.

\section{Experimental}

Fig. 1 illustrates the experimental setup of laser dicing sapphire substrate. A $532 \mathrm{~nm}$ Diode Pumped Solid State (DPSS) laser (Lightwave Series 210G) with a pulse duration of 45 ns and laser pulse energy between 0 and $650 \mu \mathrm{J}$ is used as light source. The laser beam goes through a beam attenuator to tune the pulse laser energy, followed by a beam expander to enlarge the beam diameter. The laser beam is reflected by a $45^{\circ}$ reflective mirror and then through a focus lens to ensure the focal plane is identical with the upper surface of the substrate. Its focal length is 50 $\mathrm{mm}$ and a focal beam diameter of $16 \mu \mathrm{m}$ can be achieved. A coaxial CCD camera is used to visualize and monitor the position of the laser beam in real time. The shutter and the 
translations in the $\mathrm{x}, \mathrm{y}, \mathrm{z}$ directions and the rotation in the $\mathrm{x}-\mathrm{y}$ plane are controlled by the computer generated signals.

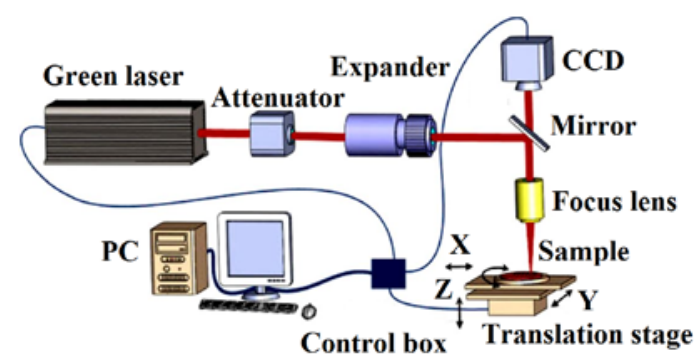

Fig. 1 Schematic diagram of the experimental setup.

Single factor method is applied in the laser dicing experiment to investigate the effect of laser scanning velocity, laser pulse energy and pulse repetition rate on the micro-morphologies of the laser-cut kerf. During the multiple pulses laser dicing experiment, the laser focal point is fixed $50 \mu \mathrm{m}$ under the sapphire surface. A singleside polished mono-crystal sapphire $\left(\mathrm{a}-\mathrm{Al}_{2} \mathrm{O}_{3}\right)$ substrate with (0001) orientation is used in the experiment. The specimen dimensions are $432 \mu \mathrm{m} \times 2$ inch (thickness $\times$ diameter), which are commercially available. All the specimens are carried out by laser micromachining without any assist gas and the specimens are cleaned by $2 \mathrm{~mol}$ $\mathrm{KOH}$ solution(20\% mass fraction) and then ultrasonically cleaned by deionized water both for 5 minutes to remove debris and contaminants. After cleaning, the sapphire substrate is separated along the ablated groove with splinter forceps. The kerf depth and width, stripes length and space are measured by an optical microscopy (ZxissAX10), meanwhile the micro-morphology of the kerf is observed by the optical microscopy and environmental scanning electron microscope (FEI Quanta 400FEG).

\section{Results and discussion}

\subsection{Experimental results}

Fig. 2 shows the SEM images of laser-cut kerf at the pulse energy of $400 \mu \mathrm{J}$, scanning speed of $1 \mathrm{~mm} / \mathrm{s}$ and pulse repetition rate of $1 \mathrm{kHz}$. It can be clearly seen from the Fig. 2 a) that some undulations stripes and three-fold cracks can be found on the bottom of the kerf. The undulations stripes are formed by recoil pressures and material resolidification during laser ablation. And the cracks imply the generation of rhombohedral twinning during multiple pulses laser processing [15]. Fig.2 b) illustrates section parallel to cut kerf, and some periodical stripes which cannot be seen from the top view are found. As a result, multiple pulses laser scanning with the focal point under the surface not only generates the surface damage, but also induces inner periodical structures.

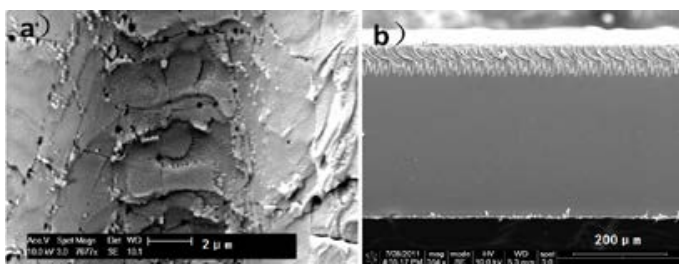

Fig. 2 a) Top view and b) Cross-section view of the periodic block stripe in the laser-cut kerf.
The periodical stripes on the sidewall are smooth and shiny without the phenomenon of resolidification. Both sides of the sidewall have the stripes, and the distance between the neighboring cupped stripes is about $10 \mu \mathrm{m}$, much larger than the distance between the neighboring two pulses (about $1 \mu \mathrm{m}$ by calculation). Therefore, the formation of the periodical stripes maybe the composite action of multiple pulses, but not single pulse ablation or damages from separation process. Because the laser ablation effect is exerted on the surface, the periodical stripes are usually covered by the resolidification layer and cannot be seen under the top view.

\subsection{Discussion}

\subsubsection{The formation of the periodical stripes on the kerf sidewall}

When the focal laser is irradiated the sapphire, the thermal temperature at the surface can be expressed by $[11,16]$

$$
\begin{array}{r}
T=T_{0}+\frac{2 A \times I_{0}}{K}\left(\frac{D \tau}{\pi}\right)^{\frac{1}{2}}\left(t \leq t_{s}\right) \\
T=\frac{2 A \times I_{0}}{K}\left(\sqrt{\frac{D t}{\pi}}-\sqrt{\frac{D\left(t-t_{f}\right)}{\pi}}\right)\left(t \geq t_{s}\right)
\end{array}
$$

Where $T_{0}$ is the room temperature, $\mathrm{A}$ is the absorption coefficient (the value is $0.03 \mathrm{~cm}^{-1}$ ) [17], $\tau$ is the pulse duration.

The laser power densityis $I_{0}$ calculated by

$$
I_{0}=\frac{4 E \times F}{\pi \times d^{2}}
$$

The thermal diffusivity $\mathrm{D}$ is also given by

$$
D=K /\left(\rho C_{P}\right)
$$

Where $E$ is the laser pulse energy, $d$ is the focal beam diameter (the value is $16 \mu \mathrm{m}$ ), $K$ is the thermal conductivity (the value is $0.4 \mathrm{~W} / \mathrm{cm} \cdot \mathrm{K}$ ), $\rho$ is the sapphire density of $3.98 \mathrm{~g} / \mathrm{cm}^{3}$, and $C_{p}$ is the specific heat of $0.85 \mathrm{~J} / \mathrm{g} \cdot \mathrm{K}$. Take the experiment data in Fig. 4 for example, when the laser pulse energy is $400 \mu \mathrm{J}$, and the room temperature $T_{0}$ is $25^{\circ} \mathrm{C}$, the calculated value of instant temperature when laser irradiates sapphire is $27610 \mathrm{~K}$, which is much higher than the sapphire boiling point of $3253 \mathrm{~K}$. Therefore, the sapphire on the focus point will vaporizes instantaneously when laser absorption begins suddenly inside the substrate.

Fig. 3 shows the schematic diagram of laser induced stripes on the kerf sidewall of sapphire substrate. When laser irradiates on the sapphire surface, the region where the temperature rises beyond $3253 \mathrm{~K}$ will be instantaneously vaporized and a void is formed. Then a thermal shock wave propagates toward the upper surface, and the high dislocation density layer is formed [18]. When the thermal shock wave pushes up the high dislocation density layer which occurred in the previous pulse irradiation, a crack whose initiation is a dislocation is produced. From our previous study [19], the thermal stress is much larger than the material strength. After the irradiation of a series of laser pulses with high overlap rates, the high strength thermal stress will generate new cracks continuously and expand 
the previous ones. And this phenomenon becomes a cycle period at last.

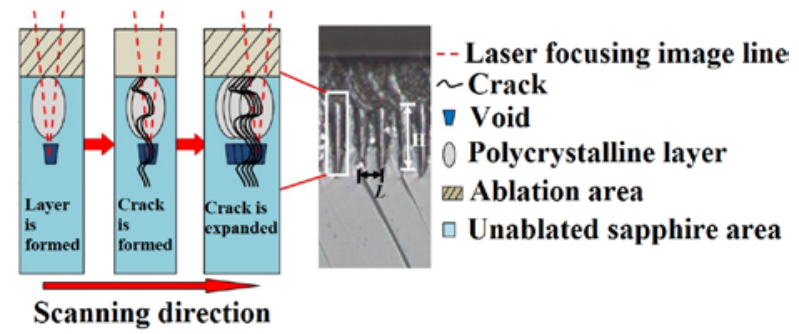

Fig.3 The schematic diagram of laser induced stripes on the kerf sidewall of sapphire substrate.

The region where the temperature rises beyond 10,000 $\mathrm{K}$ will be instantaneously vaporized and a void is formed. After that high temperature area expands rapidly in the surface direction as shown in Fig.4 . Very large temperature difference occurs between the high temperature region by laser absorption and its circumference as understood from Fig.4 . Because thermal expansion of the high temperature region is immobilized by the neighboring low temperature region, the high temperature region must receive very strong compression.

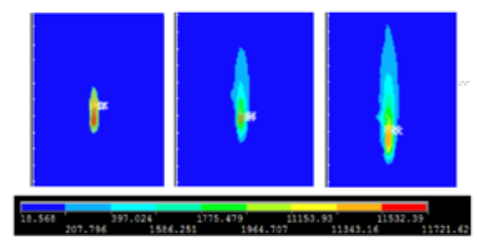

Fig.4 Time variation of temperature distribution.

The laser absorption suddenly begins at the surface once the thermal shock wave reaches the surface. Because the heat in the neighborhood of the surface is diffused only in the inside of the lower half, the surface temperature becomes very high and is maintained comparatively for a long time. As a result, not only an inside modified layer is generated in the sapphire substrate, but also the sapphire surface is removed in such a high-temperature state. And with the thermal stress loads at two points of the voids, the voids are expanded by alternating tension and compression stress. Not only the ablation zone, but also the cracks experience an increase when the laser pulse energy becomes higher. When varying the scanning velocity, laser pulse energy and laser pulse repetition rate, the variation of thermal distribution lead to the changing of stripe shapes.

When the laser beam is focused on material with high energy, strong evaporation occurs on the melt surface. The recoil pressure associated with energetic evaporation is sufficient to produce a deep, narrow depression in the keyhole.

High temperature region of about $2000 \mathrm{~K}$ propagates in the direction of the laser irradiation from the vicinity of the focal point as a thermal shock wave. The region where the thermal shock wave propagates becomes a high dislocation density layer due to the very large compressive stress.

As a result, a train of the high dislocation density layer and void, which look like a line of exclamation marks, is generated as a belt in the laser scanning direction as shown schematically in Fig.1 . When the thermal shock wave caused by the next laser pulse propagates through part of the high dislocation density layer produced by previous laser pulse, a crack whose initiation is a dislocation progresses Cracks progress from the high dislocation density region to the monocrystal area.

Laser absorption suddenly occurs in the focal point vicinity, and a void is generated. Then a thermal shock wave propagates toward the upper surface, and the high dislocation density layer is formed. When the thermal shock wave pushes up the high dislocation density layer which occurred in the previous pulse irradiation, a crack, whose initiation is a dislocation, is produced.

\subsubsection{Effect of laser processing parameters on periodi- cal stripes of kerf sidewall}

To further analyze the relationship between periodical stripes and the laser output parameters, different combinations of scanning velocity, laser pulse repetition rate and laser pulse energy are used. The stripe length $H$ and stripe space $L$ shown in Fig. 3 are also measured.

Fig. 5 shows the stripe morphologies of the kerf sidewall under different laser pulse energies. The ablation zone becomes deeper when the laser pulse energy increases, but the laser pulse energy slightly affects the space and length of the stripes. The space $L$ and the length $H$ are respectively $23 \mu \mathrm{m}$ and $42 \mu \mathrm{m}$ at the scanning velocity of $0.3 \mathrm{~mm} / \mathrm{s}$ and the laser pulse repetition rate of $1 \mathrm{kHz}$. Additionally, the laser pulse energy rarely affects the morphologies of the stripes. The neighboring stripes stay close with each other with one of them has a little longer $L$ than the other one.

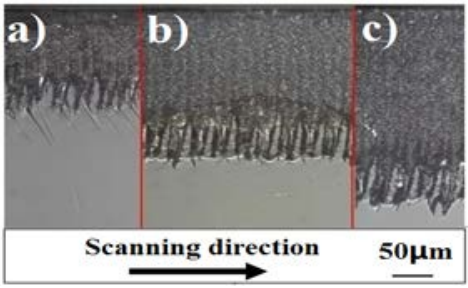

Fig. 5 Stripe morphologies of the kerf sidewall vary with the pulse energy of a) $150 \mu \mathrm{J}$, b) $400 \mu \mathrm{J}$ and c) $650 \mu \mathrm{J}$ when the scan-

ning velocity is $0.3 \mathrm{~mm} / \mathrm{s}$ and the repetition rate is $1 \mathrm{kHz}$.

Fig. 6 shows the stripe morphologies of the kerf sidewall under different scanning velocities. Significant changes occur on the stripe space $L$, stripe length $H$ and stripe shape when varying the scanning velocity. The stripe space $L$ increases and the stripe length $H$ decreases when increasing the scanning velocity. Meanwhile, the stripes with needlelike shape transform into cone-shape when increasing the scanning velocity.

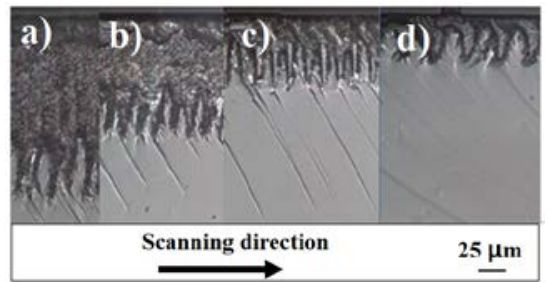

Fig.6 Stripe morphologies of the kerf sidewall vary with a scanning velocity of a) $0.3 \mathrm{~mm} / \mathrm{s}$, b) $0.5 \mathrm{~mm} / \mathrm{s}$, c) $1 \mathrm{~mm} / \mathrm{s}$ and d) 2 
$\mathrm{mm} / \mathrm{s}$ when the laser pulse energy is $150 \mu \mathrm{J}$ and the repetition rate is $1 \mathrm{kHz}$.

Moreover, the effects of laser pulse energy and scanning velocity on the stripe space $L$ and length $H$ are shown in Fig.7 . When the laser pulse repetition rate is fixed in 1 $\mathrm{kHz}$, it is obvious that when the scanning velocity is lower than $1 \mathrm{~mm} / \mathrm{s}$ the value of stripe space is nearly the same, but significant change on stripe space occurs when scanning velocity rises to $2 \mathrm{~mm} / \mathrm{s}$. The stripe length increases with the decreasing of the scanning velocity. It means the scanning velocity plays a predominant role on the stripe length. The reason is that the slow velocity leads to the increase of the laser-material interaction time and absorbed energy, and consequently more concentrated thermal stress is generated in the substrate which may lead to the narrow stripe space and deep stripe length. Although the laser pulse energy significantly affects the depth of the ablated zone, it rarely alters the stripes morphology. It is because the increasing kerf depths will reduce the laser energy reaching to the bottom of the kerf to a certain extent.
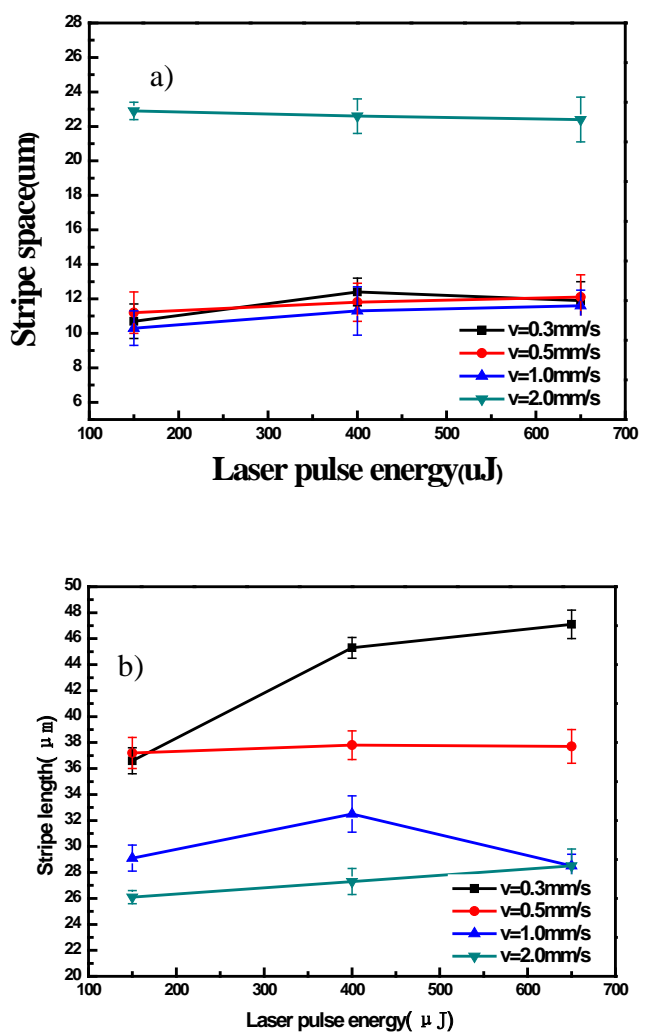

Fig. 7 a) Stripe space and b) stripe length vary with the laser pulse energy at different scanning velocities.

Fig.8 shows stripe morphology of the kerf sidewall under different laser pulse repetition rate. When the laser pulse energy is $150 \mu \mathrm{J}$, the scanning velocity is $0.5 \mathrm{~mm} / \mathrm{s}$ and the laser pulse repetition rate is $1 \mathrm{kHz}$, the stripes morphology on the sidewall are intensive and short. When the laser pulse repetition rate increases, both stripe length $H$ and space $L$ increase. The stripe morphology transforms from an intensive and short needlelike shape into a loose and long needlelike shape. And the depth of the ablated zone also increases remarkably when the laser pulse repetition rate increases from $1 \mathrm{kHz}$ to $5 \mathrm{kHz}$, but the ablated zone depth remains stable when laser pulse repetition rate increases from $5 \mathrm{kHz}$ to $10 \mathrm{kHz}$.

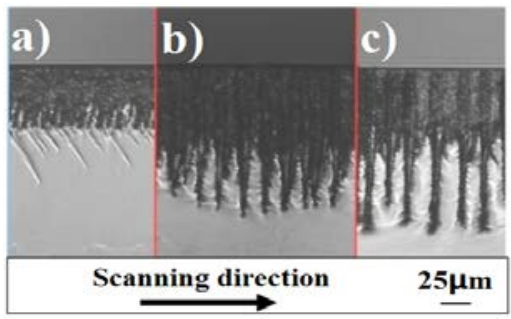

Fig.8 Stripe morphologies of the kerf sidewall vary with a laser pulse repetition rate of a) $1 \mathrm{kHz}$, b) $5 \mathrm{kHz}$ and c) $10 \mathrm{kHz}$ when the laser pulse energy is $150 \mu \mathrm{J}$ and the scanning velocity is $0.5 \mathrm{~mm} / \mathrm{s}$.

Fig.9 shows the combined effect of laser pulse repetition rate and scanning velocity on the stripe space Land stripe length $H$. From Fig. 9a) we can see that high laser pulse repetition rate and scanning velocity will lead to a wider stripe space $L$. The stripe space $L$ varies from 11.8 to $26 \mu \mathrm{m}$ with an increment of $14.2 \mu \mathrm{m}$. Fig. 9b) shows the stripe length $H$ grows with the increasing of laser pulse repetition rate. It is obvious that parameters of laser pulse repetition rate and scanning velocity have predominant effects on the stripe shapes, since both of them affect the heat accumulation per unit time in the substrate.
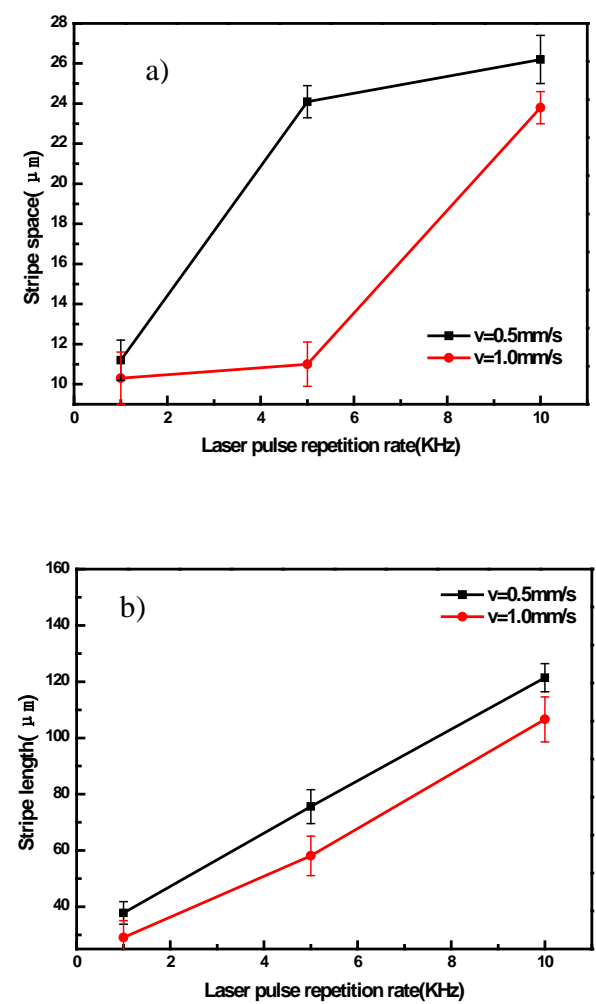

Fig. 9a) Stripe space and b) stripe length vary with the laser pulse repetition rate at different scanning velocities when laser pulse energy is of $150 \mu \mathrm{J}$

From above parameters analysis, kerf depth could be increased by inducing periodical stripes with suitable selec- 
tion of laser processing parameters. The scanning velocity and the laser pulse repetition rate are the most effective two main parameters that can affect the stripe space, stripe length and stripe shape. Laser pulse energy is the least effective one on controlling the stripes generation. Under the laser processing parameters combination of $150 \mu \mathrm{J}$ laser pulse energy, $5 \mathrm{kHz}$ laser pulse repetition rate and 0.5 $\mathrm{mm} / \mathrm{s}$ scanning velocity, the periodical stripes enlarges the cut kerf depth for about $40 \mu \mathrm{m}$.

The scribing and breaking method requires the cut kerf depth of at least $1 / 4-1 / 3$ of the substrate thickness, namely, 108-143 mm here. Furthermore, the deeper the cut kerf depth is, the easier the substrate is separated into dies in the following breaking procedure. Therefore, it is deduced that the generation of the stripes can add more on the increasing kerf depth and is favorable to breaking the sapphire substrate.

\section{Conclusion}

Periodical stripes on the bottom of sidewall of a $532 \mathrm{~nm}$ green laser-cut kerf have been found. The theoretical analysis indicates the formation of the periodical stripes is due to the multiple thermal accumulation and thermal shocks during laser dicing. The relationship between periodical stripes and processing parameters has been explored. The space $\mathrm{L}$ and length $\mathrm{H}$ of the periodical stripes are significantly related to the laser scanning velocity and laser pulse repetition rate, but are slightly affected by the laser pulse energy. The appearance of the stripes can add more on the increasing depth and is favorable to break the sapphire substrate.

\section{Acknowledgments and Appendixes}

Financial assistance for this work is granted by the National Nature Science Foundation of China (No.51575114, 50805027), Guangzhou Science and Technology Project (No. 201607010156), Guangdong Natural Science Foundation (No.S2013010014070) and Ordinary University Characteristics Innovation Project of Guangdong Province (Natural Science,No.2014KTSCX059), Open Fund of Jiangsu Key Laboratory of Precision and Micromanufacturing Technology.

\section{References}

[1] J. Han, C. Li, M. Zhang, H. Zuo, S. Meng: Optics \& Laser Technology., 41, (2009) 339.

[2] M. S. Akselrod, F. J. Bruni: J. Cryst. Growth., 360, (2012) 134.

[3] S. Nisar, M. Sheikh, Li. L: Optics \& Laser Technology., 42, (2010) 1022.

[4] I. Choudhury, S. Shirley: Optics \& Laser Technology., 42, (2010) 503.

[5] W. Hu, Y. C. Shin, G. B. King: J. Manuf. Sci. Engin., 132, (2010) 321.

[6] A. K. Dubey, V. Yadava: J. Mater. Process. Tech., 195, (2008) 15.

[7] Y. Zhang, H. Xie, H. Zheng, T. Wei, H. Yang, J. Li, X. Yi, X. Song, G. Wang, J. Li: Opt. Express., 20, (2012) 6808.

[8] X. Z. Xie, Z. Y. Pan, X. Wei, F. M. Huang, W. Hu, M. H. Hong: J. Laser Micro/Nanoengin., 6, (2011) 209.

[9] Z. Q. Huang, M. H. Hong, T. B. M. Do, Q. Y. Lin: Appl. Phys. A., 93, (2008) 159.
[10]S. Juodkazis, K. Nishimura, H. Misawa: Appl. Surf. Sci., 253, (2007) 6539.

[11]C. Q. Li, W. L. Wu, H. B. Zuo: J. Synthetic. Crystals., 39, (2010) 997.

[12]L. Qi, K. Nishii, M. Yasui: Opt. Laser. Eng., 48, (2010) 1000.

[13]M. Kumkar, L. Bauer, S. Russ, M. Wendel, J. Kleiner, D. Grossmann, K. Bergner, S. Nolte: SPIE LASE International Society for Optics and Photonics, San Diego, 115, (2014) 8972.

[14]E. Ohmura, Y. Kawahito, K. Fukumitsu, J. Okuma, H. Morita: J. Mat. Sci. Engin. A., 1, (2011) 46.

[15]S. N. Luo, P. D. Peralta, C. Ma, D. L. Paisley, S. R. Greenfield, E. N. Loomis: Appl. Surf. Sci., 253, (2007) 9457.

[16]B. S. Yilbas, M. Sami, A. Farayedhi: ARCHIVE Proceedings of the Institution of Mechanical Engineers Part C Journal of Mechanical Engineering Science., 212, (1998) 141.

[17]E. R. Dobrovinskaya, L. A. Lytvynov, V. Pishchik: Application., 27, (2009) 42.

[18]E. Ohmura, M. Kumagai, M. Nakano, K. Kuno, K. Fukumitsu, H. Morita: J. Adv. Mech. Des. Syst., 2, (2008) 540.

[19]X. Z. Xie, X. D. Huang, W. F. Chen, X. Wei, W. Hu, R. H. Che: Chinese Journal of Lasers., 40, (2013) 104.

(Received: June 21, 2016, Accepted: March 8, 2017) 